\title{
Possible risk factors for the development of sodium-glucose co-transporter 2 inhibitor- associated diabetic ketoacidosis in type 2 diabetes
}

\author{
KATE WEST, ${ }^{1}$ LUCY-ANNE WEBB,${ }^{1}$ MATTHEW FENECH ${ }^{2}{ }^{2}$ KETAN DHATARIYA ${ }^{1,2,3}$
}

\begin{abstract}
Background: In the summer of 2015 the Food and Drug Administration and European Medicines Agency reported that over 100 cases of diabetic ketoacidosis (DKA) had been identified and issued a warning of increasing concerns of a possible link between sodium-glucose co-transporter 2 (SGLT-2) inhibitor use and the development of DKA. Several mechanisms for this have been postulated, but precisely what factors predispose individuals to develop DKA remain unknown.

Methods: We conducted a literature search on Pubmed and Ovid databases and Google Scholar and report a further case of DKA occurring in a patient with a 16-year history of type 2 diabetes 10 months after starting dapagliflozin.

Results: Very few cases of DKA occurring in type 2 diabetes have been reported. Most cases of DKA have occurred in patients with type 1 diabetes taking part in clinical trials or given the drug 'off licence'. Several potential mechanisms for developing DKA have been postulated. In addition to these, we suggest that a prolonged history of type 2 diabetes may lead to reduced beta cell reserve, increasing the probability of developing DKA.

Conclusion: Despite the insulin-independent mode of action, SGLT-2 inhibitors are currently only licensed for use in type 2 diabetes. If the duration of diabetes is long and/or if insulin doses are reduced on initiation, clinicians and patients should be wary of signs of DKA. Until further
\end{abstract}

Department of Medicine, Norfolk and Norwich University Hospitals NHS Foundation Trust, Norwich, UK

Norwich Medical School, University of East Anglia, Norwich Research Park, Norwich, UK

Elsie Bertram Diabetes Centre, Norfolk and Norwich University Hospitals NHS Foundation Trust, Norwich, UK

Address for correspondence: Dr Ketan Dhatariya Consultant in Diabetes and Endocrinology, Diabetic Foot Clinic, Elsie Bertram Diabetes Centre, Norfolk and Norwich University Hospitals NHS

Foundation Trust, Colney Lane, Norwich, Norfolk NR4 7UY, UK

Tel: +44 (0)1603 288170 Fax: +44 (0)1603 287320

E-mail: ketan.dhatariya@nnuh.nhs.uk

http://dx.doi.org/10.15277/bjd.2016.079 data are available, unlicensed use in type 1 diabetes should be avoided.

Br J Diabetes 2016;16:78-81

Key words: SGLT-2 inhibitors, diabetic ketoacidosis

\section{Introduction}

In the summer of 2015 the US Food and Drug Administration (FDA) and the European Medicines Agency (EMA) issued warnings about the risk of developing diabetic ketoacidosis (DKA) in patients on sodium-glucose co-transporter 2 (SGLT-2) inhibitors. ${ }^{1,2}$ The EMA website mentioned that they have had over 100 cases reported to them. Two recent publications have summarised the data to date. ${ }^{3,4}$ However, what is clear from these publications is that a large number of cases have been seen in clinical trials for SGLT-2 inhibitor use in people with type 1 diabetes (T1DM). From a pharmacological point of view, these trials make sense, given the insulin-independent mode of action of these agents. However, only a very small minority (2 out of the 13 reported by Peters et $a^{\beta}$ ) are in people with type 2 diabetes (T2DM). In addition, pooled data from canagliflozin trials suggested that only 12 out of 17,596 patients developed DKA; however, these data have been questioned and may be a gross underestimate. ${ }^{6}$ Thus, if a person with T1DM is being prescribed these drugs (off licence) but then develops DKA, they will need to be prepared to face the potential consequences. If more people with T2DM develop DKA, then the pharmaceutical industry needs to take notice.

\section{Methods}

We conducted a literature search on the Pubmed and Ovid databases and Google Scholar using the search terms ("DKA" [All Fields] OR "Diabetic ketoacidosis" [All Fields] OR "Ketosis" [All Fields] OR "Ketoacidosis" [All Fields]) AND ("SGLT-2 inhibitors" [All Fields] OR "Sodium glucose co-transporter" [All Fields]). Several mechanisms have been postulated and are shown in Box 1. The data that have been published do not give any great detail of the patients, only the potential mechanisms.

Results

Table 1 shows the results of this search for people with T2DM and the case described below. Of these, two cases had undiag- 
Box 1 Postulated mechanisms to allow the development of diabetic ketoacidosis in patients on SGLT-2 inhibitors

- A reduction in insulin dose when insulin and SGLT-2 inhibitors are combined

- This lower dose of insulin may be insufficient to suppress lipolysis and ketogenesis

- SGLT-2 inhibitors promote glucagon secretion

- SGLT transporters decrease urinary excretion of ketone bodies. A decrease in renal clearance of ketone bodies could thus increase plasma ketone (in particular beta hydroxybutyrate) concentrations

- SGLT-2 inhibitors are associated with mild dehydration, potentially exacerbating the ketonaemia

- Progressive beta cell decline with age meaning there is less reserve

nosed pancreatic insufficiency ${ }^{7}$ and one reported a $25.4 \mathrm{~kg}$ weight loss in the 6 months prior to starting the SGLT-2 inhibitor, suggesting potentially undiagnosed T1DM. ${ }^{8}$ The last case described a patient who followed a strictly reduced carbohydrate diet in the time period leading up to presentation with DKA. ${ }^{9}$

\section{Case report}

In May 2015 a 48-year-old woman with a 16year history of T2DM and hypertension presented to the emergency department with a 4-week history of back pain and malaise. She had no other medical history and denied alcohol use. For the 4 days prior to admission she had been experiencing increasing polyuria and polydipsia. She had been vomiting and therefore had been unable to eat and drink.

After her initial diagnosis of T2DM in 1999, she had been treated with diet and lifestyle changes for 5 months. However, her glycaemic control remained suboptimal and she was started on metformin. Over the following 5 years the dose of metformin was increased and additional agents had been added to her regimen, including gliclazide and exenatide, both of which were increased to maximal doses. Despite these interventions, her weight continued to rise and her glycaemic control remained suboptimal. In October 2014, because she had failed to lose weight, exenatide was stopped and dapagliflozin was started. The patient reported she lost $20 \mathrm{~kg}$ over the subsequent 10

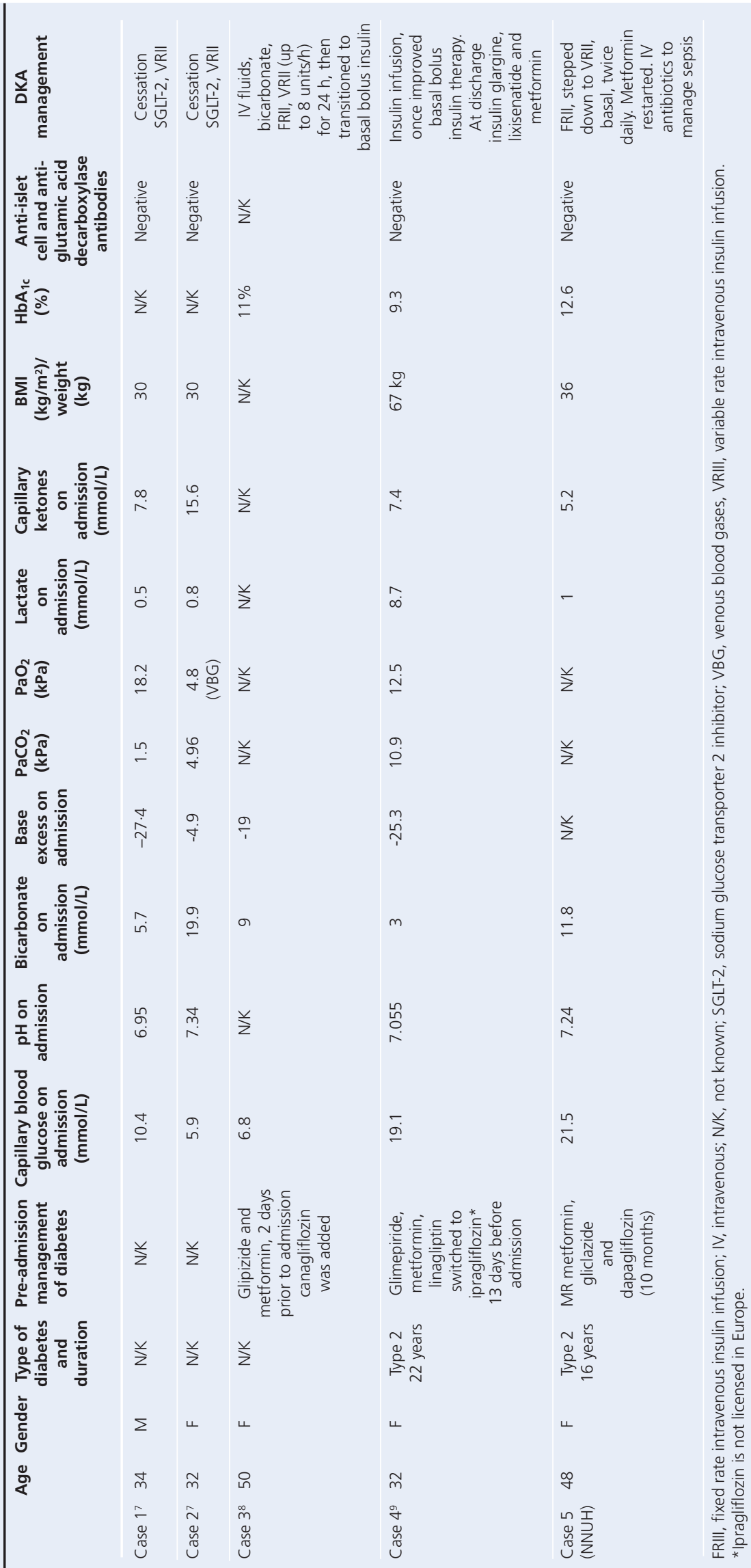


months. When she commenced dapagliflozin her glycated haemoglobin $\left(\mathrm{HbA}_{1 \mathrm{c}}\right)$ had been $112 \mathrm{mmol} / \mathrm{mol}(12.4 \%)$ and was $114 \mathrm{mmol} / \mathrm{mol}(12.6 \%) 2$ months prior to admission. On admission to hospital her medication was metformin, gliclazide, dapagliflozin, losartan and ezetimibe. For back pain she was taking naproxen, tramadol and amitriptyline.

On presentation the patient had a blood glucose concentration of $21.5 \mathrm{mmol} / \mathrm{L}$ (NR $3.5-7.8 \mathrm{mmol} / \mathrm{L}$ ), capillary ketone concentration of $5.2 \mathrm{mmol} / \mathrm{L}(<0.1 \mathrm{mmol} / \mathrm{L})$, a venous $\mathrm{pH}$ of 7.24 and serum bicarbonate of $11.8 \mathrm{mmo} / \mathrm{L}(22-30 \mathrm{mmol} / \mathrm{L})$ with a lactate of $1.0 \mathrm{mmol} / \mathrm{L}$ suggestive of DKA. Inflammatory markers were raised with a white cell count of $19.5 \times 10 \% / \mathrm{L}$ (4.0$\left.11.0 \times 10^{9} / \mathrm{L}\right)$ and C-reactive protein $577 \mathrm{mg} / \mathrm{L}(0-10 \mathrm{mg} / \mathrm{L})$. Her renal function was normal, with a calculated anion gap of 32 $\mathrm{mmol} / \mathrm{L}$ (3-11 mmol/L). Alcohol, salicylate and paracetamol levels were undetectable. Her anti-islet cell antibody and anti-glutamic acid decarboxylase antibody were both negative. Insulin and C-peptide results were unavailable.

She was treated in accordance with the UK national DKA guideline. ${ }^{10}$ Metabolic correction was slower than expected and only after $24 \mathrm{~h}$ following admission was she able to resume normal oral intake. Her metformin was then restarted and she was initiated on biphasic human insulin.

\section{Discussion}

A systematic review of SGLT-2 receptor inhibitors reported significant reductions in $\mathrm{HbA}_{1 \mathrm{c}}$, body weight, blood pressure and incidence of hypoglycaemia. ${ }^{11}$ Thus, their use has increased in the hope that improvements in these surrogate markers for premature cardiovascular disease will translate into lower mortality. However, as part of these studies and anecdotally, the numbers of people reported with DKA on these agents has increased, with enough numbers for the FDA to issue a warning about this association. ${ }^{1}$

Because of the largely insulin-independent mode of action, the mechanism underpinning an association between SGLT-2 inhibitor use and DKA remains unclear, although more evidence is emerging. ${ }^{4}$

Animal data show that the use of these agents can stimulate ketogenesis and, at the same time, reduce their renal excretion. ${ }^{12}$ In humans there are data to show that fatty acid oxidation and ketone body production increases on initiation of these drugs. ${ }^{13,14}$ Furthermore, on starting an SGLT-2 inhibitor, the resultant decreased circulating glucose, in association with the patient's insulinopenia or insulin resistance, results in decreased glycogen synthesis. ${ }^{15}$ Glycogen synthesis is further compromised because SGLT-2 inhibitors increase gluconeogenesis and serum glucagon levels.7,16 The use of these agents leads to a relative hyperglucagonaemia, and it is the glucagon response to frank hypoglycaemia that is diminished. This relative hyperglucagonaemia promotes lipolysis, and if this is of sufficient magnitude and/or sufficiently prolonged (in the presence of insufficient insulin), this will give rise to the excess fatty acid metabolism diverting to ketones. It may be hypothesised that, during acute intercurrent illness, the combination of increased insulin resist- ance (compared with the patient's baseline in T2DM) and depleted glycogen stores may result in increased lipolysis and fatty acid metabolism, resulting in ketogenesis. Indeed, in previously reported cases it is possible to see how pancreatic insufficiency may make this hypothesised depletion in glycogen reserves more likely to occur.? In addition, a patient with pancreatic insufficiency may also have depleted alpha cell numbers resulting in reduced levels of glucagon in response to hypoglycaemia. ${ }^{15}$ This would drive further lipolysis and ketogenesis, potentially producing a picture of euglycaemic DKA. Similarly, a reduced carbohydrate diet or recent significant weight loss in combination with SGLT-2 inhibitor use may also predispose to reduced glycogen reserves, reliance of free fatty acid metabolism and early ketogenesis. ${ }^{15}$

Progressive beta cell failure, potential misdiagnosis of T1DM as T2DM, inappropriate reduction in insulin dose on initiation of the SGLT-2 inhibitor and the resultant insulinopenia may all play a significant role in the association seen between SGLT-2 inhibitors and DKA. It has previously been suggested that beta cell function declines with increasing duration of T2DM. ${ }^{17}$ More recent work suggests that this may be reduced by up to $40 \% 4$ years after diagnosis. ${ }^{18}$ Currently, SGLT-2 inhibitors are included as 'second line treatment' for T2DM. ${ }^{19}$ However, it is likely that they are currently introduced several years after the diagnosis is made, when other hypoglycaemic agents have failed and hence when the residual beta cell reserve is likely to be low. One of the limitations of studies of SGLT-2 inhibitors is that no analysis has been done on the effectiveness of these drugs after different durations of diabetes, nor whether this is a factor in determining the risk of developing DKA. ${ }^{9}$ Hayami et al also hypothesised that this class of oral hypoglycaemic agent may be particularly useful in patients who have had diabetes for a longer duration in whom sulphonylureas have become less effective due to reduced beta cell activity. ${ }^{9}$ However, we would postulate the opposite: if an SGLT-2 inhibitor is to be used, it should be early where beta cell reserve function is retained. The lack of data reporting the duration of diabetes in those who have developed DKA means that this postulated mechanism remains speculative.

With the increasing numbers of DKA being reported in patients on these agents, clinicians should be aware of some risk factors. These include a faster than predicted weight loss because this may indicate reduced beta cell function. ${ }^{1,9}$ We also suggest immediate cessation of the medication if the patient becomes acutely unwell or has reduced oral intake. Finally, in the acute management of possible SGLT-2 inhibitor-induced DKA, the increased glycosuria has been shown for up to 11 days following cessation of the SGLT-2 inhibitor, ${ }^{6}$ meaning that metabolic correction may be slower than expected.

In summary, although recent data from the empagliflozin cardiovascular safety study have shown a reduction in cardiovascular and all-cause mortality in people with $\mathrm{T}_{2} \mathrm{DM}, 20$ it is perhaps unsurprising that inhibition of a process of central import to energy homeostasis in health may result in previously unforeseen effects on carbohydrate and lipid metabolism. We suggest that it is those patients who have low insulin levels - either due to 


\section{Key messages}

- SGLT-2 inhibitors work in an insulin independent manner to improve glycaemic control, but the reduction in insulin dose may contribute to the development of ketoacidosis

- DKA with SGLT-2 inhibitor use has most often been reported in the trials using it in people with type 1 diabetes, but vigilance must be maintained because it can also occur in those with type 2 diabetes

- The same metabolically stressful events that that cause DKA in type 1 diabetes can cause it in SGLT-2 inhibitor associated DKA

beta cell failure or a conscious reduction in concurrently administered insulin dose - who are most at risk of this complication. Further examination of the reported cases is required. In addition, more work needs to be done to assess the roles of insulin resistance, the degree of beta cell failure and the effects of recent weight loss in the risk of developing DKA on SGLT-2 inhibitors.

Author contributions All of the authors looked after the patient. KW and L-AW carried out the literature review and wrote the initial drafts of the manuscripts. MEF edited the drafts. KD wrote the final version. All of the authors have seen and approved the final version of this manuscript.

Acknowledgements We thank the patient for giving us written permission to describe her case.

Conflict of interest KW, L-AW and MEF declare that they have no conflicts of interest. KD has accepted honoraria for speaking from manufacturers of SGLT-2 inhibitors and is on the Severe Hypoglycemia and DKA Clinical Endpoint Adjudication Committee for Sotaglifozin, for Lexicon Pharmaceuticals.

Funding KW, L-AW and KD are employees of the UK National Health Service. MEF is funded by the British Heart Foundation.

\section{References}

1. US Food and Drug Administration. FDA Drug Safety Communication: FDA warns that SGLT2 inhibitors for diabetes may result in a serious condition of too much acid in the blood. http://www.fda.gov/Drugs/ DrugSafety/ucm446845.htm. 2015. [Last accessed 18 December 2015]

2. European Medicines Agency. Review of diabetes medicines called SGLT2 inhibitors started. http://www.ema.europa.eu/docs/en_GB/document_library/Referrals_document/SGLT2_inhibitors_20/Procedure_started/WC 500187926.pdf. 2015. [Last accessed 18 December 2015]

3. Peters AL, Buschur EO, Buse JB, et al. Euglycemic diabetic ketoacidosis: a potential complication of treatment with sodium-glucose cotransporter 2 inhibition. Diabetes Care 2015;38:1687-93.

http://dx.doi.org/10.2337/dc15-0843
4. Taylor SI, Blau JE, Rother KI. Perspective: SGLT2 inhibitors may predispose to ketoacidosis. J Clin Endocrinol Metab 2015;100:2849-52. http://dx.doi.org/10.1210/jc.2015-1884

5. Erondu N, Desai M, Ways K, et al. Diabetic ketoacidosis and related events in the canagliflozin type 2 diabetes clinical program. Diabetes Care 2015;38:1680-6. http://dx.doi.org/10.2337/dc15-1251

6. Dhatariya K. Comment on Erondu $\mathrm{N}$ et al - Does this data significantly underestimate the prevalence of SGLT-2 associated DKA? Diabetes Care 2016;39(1):e18.

7. Hine J, Paterson H, Abrol E, et al. SGLT inhibition and euglycaemic diabetic ketoacidosis. Lancet Diabetes Endocrinol 2015;3:503-04. http://dx.doi.org/10.1016/S2213-8587(15)00204-1

8. Burr K, Nguyen A-T, Rasouli N. A case report of ketoacidosis associated with canagliflozin (Invokana). http://press.endocrine.org/doi/abs/ 10.1210/endo-meetings.2015.DGM.5.SAT-595. 2015. [Last accessed 18 December 2015]

9. Hayami $T$, Kato $Y$, Kamiya $H$, et al. Case of ketoacidosis by a sodiumglucose cotransporter 2 inhibitor in a diabetic patient with a low-carbohydrate diet. J Diabetes Investig 2015;6:587-90. http://dx.doi.org/10.1111/jdi.12330

10. Dhatariya K, Savage M, Kelly T, et al. Joint British Diabetes Societies Inpatient Care Group. The management of diabetic ketoacidosis in adults. Second edition. Update: September 2013. http://www. diabetologistsabcd.org.uk/JBDS/JBDS.htm. 2013 [Last accessed 18 December 2015]

11. Clar C, Gill JA, Court R, et al. Systematic review of SGLT2 receptor inhibitors in dual or triple therapy in type 2 diabetes. BMJ Open 2012; 2. http://dx.doi.org/10.1136/bmjopen-2012-001007

12. Yokono M, Takasu T, Hayashizaki Y, et al. SGLT2 selective inhibitor ipragliflozin reduces body fat mass by increasing fatty acid oxidation in high-fat diet-induced obese rats. Eur J Pharmacol 2014;727:66-74. http://dx.doi.org/10.1016/j.ejphar.2014.01.040

13. Ferrannini E, Muscelli E, Frascerra $S$, et al. Metabolic response to sodiumglucose cotransporter 2 inhibition in type 2 diabetic patients. J Clin Invest 2014;124:499-508. http://dx.doi.org/10.1172/JC172227

14. Kaku K, Watada H, Iwamoto Y, et al. Efficacy and safety of monotherapy with the novel sodium/glucose cotransporter-2 inhibitor tofogliflozin in Japanese patients with type 2 diabetes mellitus: a combined Phase 2 and 3 randomized, placebo-controlled, double-blind, parallel-group comparative study. Cardiovasc Diabetol 2014;13:65. http://dx.doi.org/10.1186/1475-2840-13-65

15. Nadel E. Metabolism and nutition. In: Medical Physiology. Boron W, Boulpaep E, eds. Philadelphia: Saunders/Elsevier, 2015, p.1230.

16. Bonner C, Kerr-conte J, Gmyr V, et al. Inhibition of the glucose transporter SGLT2 with dapagliflozin in pancreatic alpha cells triggers glucagon secretion. Nature Med 2015;21:512-17. http://dx.doi.org/10.1038/nm.3828

17. Holman RR. Assessing the potential for a-glucosidase inhibitors in prediabetic states. Diabetes Res Clin Pract 1998;40:S21-5. http://dx.doi.org/10.1016/S0168-8227(98)00038-2

18. Merovci A, Mari A, Solis C, et al. Dapagliflozin lowers plasma glucose concentration and improves B-cell function. J Clin Endocrinol Metab 2015;100:1927-32. http://dx.doi.org/10.1210/jc.2014-3472

19. Inzucchi SE, Bergenstal RM, Buse JB, et al. Management of hyperglycemia in type 2 diabetes, 2015: a patient-centered approach. Update to a position statement of the American Diabetes Association and the European Association for the Study of Diabetes. Diabetes Care 2015;38:140-9. http://dx.doi.org/10.2337/dc14-2441

20. Zinman B, Wanner C, Lachin JM, et al. Empagliflozin, cardiovascular outcomes, and mortality in type 2 diabetes. N Engl J Med 2015;373:211728. http://dx.doi.org/10.1056/NEJMoa1504720 